\title{
ELECTROMODULATION OF PHOTOLUMINESCENCE FROM CdSe NANORODS FILM
}

\author{
A. LUTICH \\ Institute of Molecular and Atomic Physics, NASB \\ Nezavisimosti Ave. 70, 220072 Minsk, Belarus \\ M. ARTEMYEV, E. USTINOVICH \\ Institute for Physico-Chemical Problems, Belarusian State University \\ Leningradskaya 14, 220080 Minsk, Belarus
}

I. M. SOGANCI, H. V. DEMIR

Department of Physics, Department of Electrical and Electronics Engineering Nanotechnology Research Center, Bilkent University

Bilkent, 06800 Ankara, Turkey

\begin{abstract}
We studied photoluminescence (PL) properties of CdSe nanorods integrated in a thin film sandwiched between transparent electrodes to which an electric field applied. Nearly $20 \%$ of PL quenching accompanied with the weak Stark shift have been observed. This effect is proposed to be used for PL modulation, in particular in the wavelength range beyond the range that traditional optoelectronic devices may cover.
\end{abstract}

\section{Introduction}

Optoelectronic nanodevices that rely on electric field effects in optical absorption and emission provide the ability to be controlled conveniently using integrated electronic platforms. Semiconductor quantum dots are theoretically expected as an excellent candidate for such optoelectronic nanomaterials to show optical properties strongly dependent on electric field [1]. In the general class of quantum dots, chemically synthesized semiconductor nanocrystals also exhibit electric field effects, for example, as demonstrated in their optical absorption (e.g. the quantum confined Stark effect [2,3]) and in their optical emission as the Stark shift and luminescence quenching $[4,5])$.

In the present paper, we investigate the influence of external electric field onto luminescence properties of chemically synthesized CdSe nanorods. Unlike quantum dots, the nanorods exhibit quantum confinement only in two dimensions. It is suggested that the electric field applied along the nanorods axis may provide an effective exciton dissociation resulting in the strong quenching of PL. 


\section{The samples}

$\mathrm{CdSe} / \mathrm{ZnS}$ core-shell nanorods with size $c a .4 \times 25 \mathrm{~nm}$ were synthesized according to published procedures [6]. The polymeric polymethylmetacrylate (PMMA) film with CdSe nanorods was placed between two transparent indiumtin oxide (ITO) electrodes sealed with epoxy glue. The electric field influence on the nanorods PL was studied by applying a constant dc voltage between transparent ITO electrodes. The PL was excited by $488 \mathrm{~nm}$ Ar-ion laser. The PL spectra at different voltages were measured by a combination of inverted microscope and liquid nitrogen (LN)-cooled CCD camera based spectrometer.

\section{Results}

Fig. 1 shows PL spectra of CdSe nanorods in PMMA film at different applied voltages.

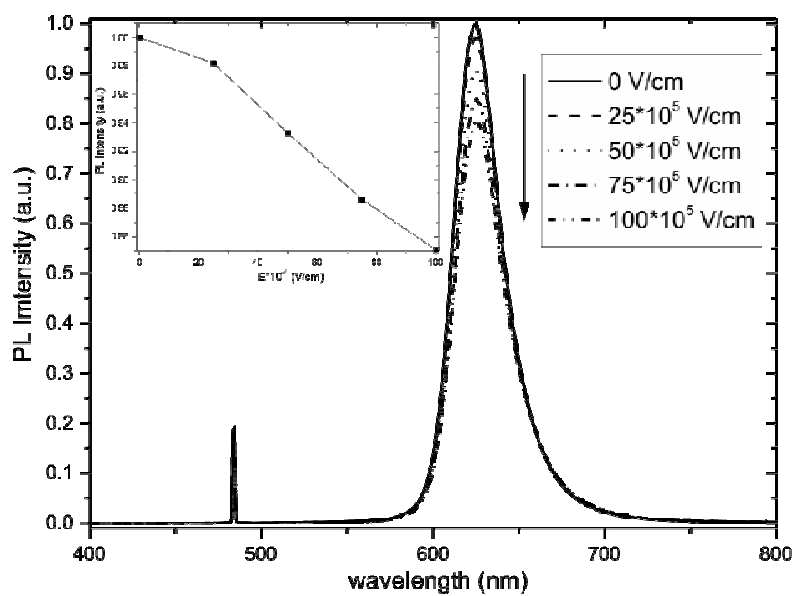

Figure 1. Photoluminescence spectra of CdSe nanorods film at different applied voltages. Inset shows the integrated PL intensity vs. the applied electric field.

There is a decrease in PL intensity (quenching) and red shift with the voltage increased. At $\mathrm{E}=10^{7} \mathrm{~V} / \mathrm{cm}$ the magnitude of PL quenching reaches $20 \%$ with respect to the signal measured with no voltage applied (Fig. 2, inset). Differential PL spectra at various electric fields applied with respect to zero field are shown in Fig. 3a. Both the negative differential PL signal at $\lambda=620 \mathrm{~nm}$ (PL quenching) and positive signal at $\lambda=670 \mathrm{~nm}$ (Stark shift) rise with the voltage increase. In order to demonstrate the Stark shift in PL spectra of the nanorods in strong electric field the differential spectra normalized to zero-field PL spectrum are presented in Fig. 3b. The character of normalized differential spectra is 
typical for those observed earlier in CdSe quantum dots ensemble and attributed to the quantum-confined Stark effect [3].

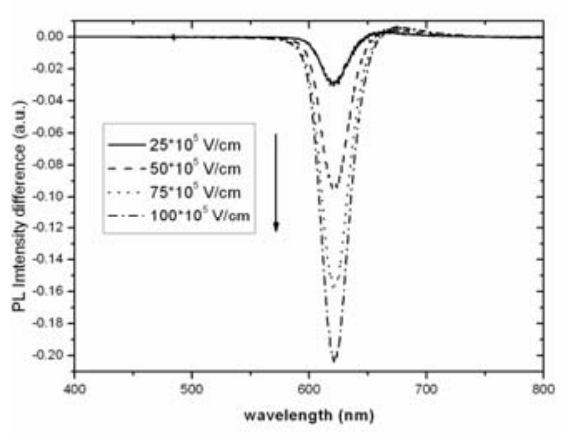

(a)

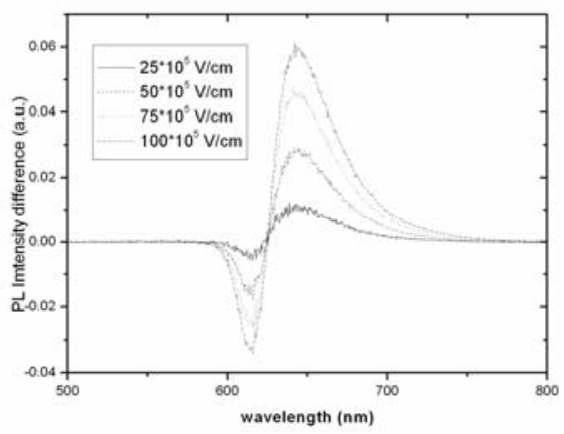

(b)

Figure 2. Differential PL (a) and normalized differential PL (b) spectra of CdSe nanorods vs. electric field applied.

\section{Conclusions}

We observed the sensitivity of PL properties of CdSe nanorods to applied electric field. This effect may find applications to make a luminescence modulator in which the incoming beam optically pumps the active part of the device (nanorods) and the resulting PL is electrically switched on and off with an applied voltage (input RF signal).

\section{Acknowledgment}

This work is partially supported by PHOREMOST Network of Excellence and INTAS (05-109-4204).

\section{References}

1. S.Gaponenko. Optical Properties of Semiconductor Nanocrystals (Cambridge University Press, 1998).

2. G.W.Wen, J.Y.Lin, H.X.Jiang, Z.Chen, Phys. Rev. B 52, 5913 (1995).

3. V.L.Colvin, K.L.Cunningham, A.P.Alivisatos, J. Chem. Phys. 101, 7122 (1994).

4. M.V.Artemyev, G.P.Yablonski, Yu.P.Rakovich, Acta Phys. Polon. A 87, 523 (1995).

5. A.W.Achtstein, H.Karla, B.Stritzker, Appl. Phys. Lett. 89, 061103 (2006).

6. T.Mokari, U.Banin, Chem. Mater. 15, 3955 (2003). 